POS PROCEEDINGS

\title{
Kaonic atoms measurements at the DAФNE Collider
}

\author{
D. Sirghi ${ }^{\star a b}$, A. Amirkhani ${ }^{c}$, A. Baniahmad ${ }^{c}$, M. Bazzi $^{a}$, G. Bellotti ${ }^{c}$, C. Berucci ${ }^{a d}$, \\ D. Bosnar ${ }^{e}$, M. Bragadireanu ${ }^{b}$, M. Cargnelli ${ }^{d}$, C. Curceanu ${ }^{a}$ A. Dawood Butt ${ }^{c}$, L. De \\ Paolis $^{a}$ R. Del Grande ${ }^{a}$, L. Fabbietti ${ }^{f}$, C. Fiorini ${ }^{c}$, F. Ghio ${ }^{g}$, C. Guaraldo ${ }^{a}$, M. Iliescu $^{a}$, \\ M. Iwasaki ${ }^{h}$, P. Levi Sandri ${ }^{a}$, J. Marton ${ }^{a d}$, M. Miliucci ${ }^{a}$, P. Moskal ${ }^{i}$, S. Niedźwiecki ${ }^{i}$, \\ S. Okada ${ }^{h}$, D. Pietreanu ${ }^{a b}$, K. Piscicchia ${ }^{a j}$, A. Scordo ${ }^{a}$, H. Shi ${ }^{a, d}$, M. Silarski ${ }^{i}$, \\ F. Sirghi ${ }^{a, b}$, M. Skurzok ${ }^{i}$, A. Spallone ${ }^{a}$, H. Tatsuno ${ }^{k}$, O. Vazquez Doce ${ }^{a f}$, \\ E. Widmann ${ }^{d}$, J. Zmeskal ${ }^{a d}$
}


INFN, Laboratori Nazionali di Frascati, Frascati (Roma), Italy

$b$

Horia Hulubei National Institute of Physics and Nuclear Engineering (IFIN-HH), Magurele, Romania

c

Politecnico di Milano, Dipartimento di Elettronica, Informazione e Bioingegneria and INFN Sezione di Milano, Milano, Italy

d

Stefan-Meyer-Institut für Subatomare Physik, Vienna, Austria

Department of Physics, Faculty of Science, University of Zagreb, Croatia

f

Excellence Cluster Universe, Technische Universiät München, Garching, Germany

g

INFN Sez. di Roma I and Inst. Superiore di Sanita, Roma, Italy

h

RIKEN, Tokyo, Japan

i

The M. Smoluchowski Institute of Physics, Jagiellonian University, Kraków, Poland

j

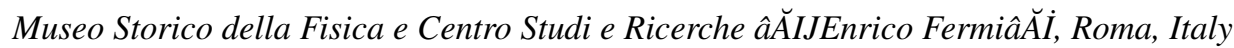

$k$

Lund Univeristy, Lund, Sweden

E-mail: sirghi@lnf.infn.it, aidin.amirkhaniepolimi.it.

ata.baniahmadepolimi.it,

massimiliano.bazzi@lnf.infn.it,giovanni.bellotti@polimi.it,

carolinaberucci@gmail.com, bosnarephy.hr,

mario.bragadireanu@nipne.ro, michael.cargnelli@oeaw.ac.at,

catalina. curceanu@lnf.infn.it, arslandawood.butt@polimi.it,

luca.depaolis@lnf.infn.it,raffaele.delgrandedlnf.infn.it,

laura.fabbietti@ph.tum.de, carlo.fiorini@polimi.it,

francesco.ghiodlnf.infn.it, guaraldodlnf.infn.it,

mihai.iliescu@lnf.infn.it, paolo.levisandri@lnf.infn.it,

masa@riken.jp, johann.marton@oeaw.ac.at,

marco.miliucci@lnf.infn.it, ufmoskal@googlemail.com,

szymonniedzwiecki@googlemail.com,sokada@riken.jp,

dorel.pietreanu@nipne.ro, kristian.piscicchia@gmail.com,

alessandro.scordo@lnf.infn.it, hexishi@lnf.infn.it,

fsirghi@lnf.infn.it, mskurzok@gmail.com,

antonio.spallonedlnf.infn.it, hideyuki.tatsuno@gmail.com,

oton.vazquez@universe-cluster.de, eberhard.widmann@oeaw.ac.at and

johann.zmeskaldoeaw.ac.at 
The DAФNE electron-positron collider of the Laboratori Nazionali di Frascati of INFN is a worldwide unique low-energy kaon source, which is being used to produce and to study kaonic atoms by the SIDDHARTA collaboration. The X-ray measurements of kaonic atoms play an important role for understanding the low-energy QCD in the strangeness sector. Significant achievements have been obtained by the SIDDHARTA experiment, among which: the most precise kaonic hydrogen measurement of the $1 s$ level shift and width induced by the presence of the strong interaction; an upper limit of the X-ray yield for kaonic deuterium $K$-series; the accurate measurement of the $2 p$ level shift and width of kaonic helium-4 and kaonic helium-3; yields of various light kaonic atoms transitions. Using the experience gained with SIDDHARTA experiment, the first kaonic deuterium measurement is in preparation in the framework of the SIDDHARTA-2 experiment, with the goal to determine the antikaon-nucleon isospin dependent scattering lengths, which is possible only by combining the $\mathrm{K}^{-} \mathrm{p}$ and the upcoming $\mathrm{K}^{-} \mathrm{d}$ results. An overview of the experimental results of SIDDHARTA and an outlook to SIDDHARTA-2 experiments are given in this paper.

XIII Quark Confinement and the Hadron Spectrum - Confinement2018

31 July - 6 August 2018

Maynooth University, Ireland

\footnotetext{
* Speaker.
} 


\section{Introduction}

A kaonic atom is formed when a negative kaon enters a target and is slowed down to a kinetic energy of a few tens of $\mathrm{eV}$ by ionisations and excitations of the molecules of the target. The entering kaon is stopped inside the target and is captured by a target atom into an outer atomic orbit, in the $\mathrm{n} \simeq 25$ excited state for kaonic hydrogen (as an example) by replacing an electron. Slowing down and capture occur on a time scale from picoseconds to nanoseconds. Then, the kaonic atom experiences a series of deexcitation processes which, at lower lying states, are dominated by X-ray emission. Finally, the kaon reaches the ground state where the strong interaction adds up to the electromagnetic one.

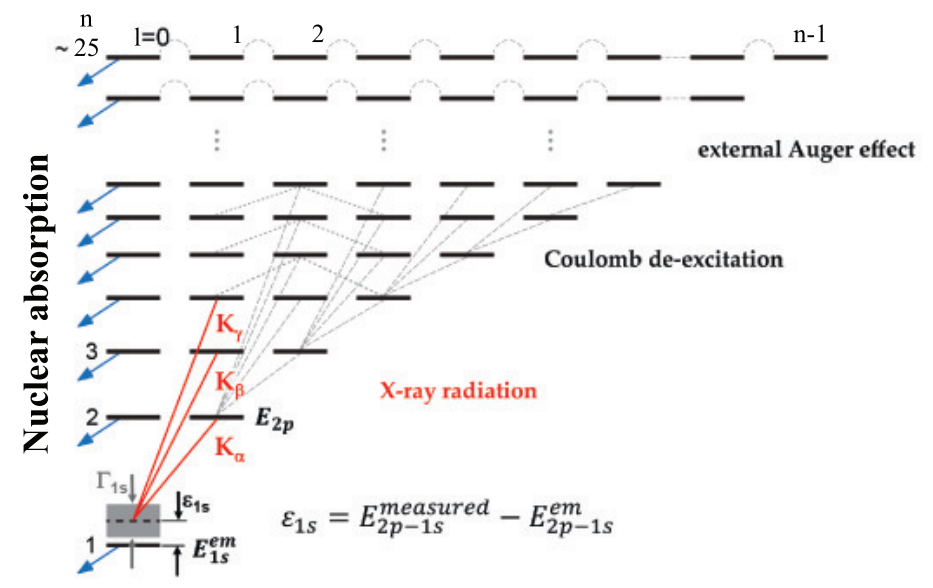

Figure 1: Cascade processes for kaonic hydrogen, starting when the kaon is captured in a highly excited state, down to the $1 s$ ground state, which is shifted due to strong interaction and broadened due to nuclear absorption of the kaon by the proton. The shift and width of the $2 p$ state due to strong interaction are negligible [1].

Since the atomic binding energies of light kaonic atoms are in the keV range, the kaonic atoms offer the unique opportunity to perform experiments equivalent to scattering at vanishing relative energy. This allows the study of the antikaon-nucleon/nucleus interaction "at threshold", without the need of extrapolation to zero relative energy. Of particular interest in kaonic atoms experiments are the studies of systems formed with hydrogen isotopes, which give access to the basic low-energy physics parameters like the antikaon-nucleon scattering lengths. Kaonic deuterium enables access to the antikaon-neutron system. Other light elements provide information on how to construct the antikaon-nucleus interaction from the elementary reactions. It is noteworthy that light exotic atoms are formed almost "electron-free", which opens the possibility for high-precision measurements, due to the absence of electron screening effect.

For the study of the strong interaction the observables of interest are the $\operatorname{shift}(\varepsilon)$ and the width $(\Gamma)$ of the atomic level, caused by the strong interaction, relative to the electromagnetic value. The electromagnetic values of the energy levels can be calculated at a precision of $\mathrm{eV}$ by solving the Klein-Gordon equation. Even a small deviation from the electromagnetic value allows to get information of the strong interaction between the kaon and the nucleus. 
In the case of kaonic hydrogen the relation between $\varepsilon_{1 s}$ and $\Gamma_{1 s}$ and the $K^{-} p$ complex scattering length $a_{K^{-}} p$ is given by the so-called Deser-Trueman formula [2,3], where the of the isospinbreaking corrections are included $[4,5]$ :

$$
\varepsilon_{1 s}+\frac{i}{2} \Gamma_{1 s}=2 \alpha^{3} \mu^{2} a_{K^{-}}\left[1-2 \alpha \mu(\ln \alpha-1) a_{K^{-}}+\ldots\right]
$$

A similar formula holds for the $K^{-} d$ scattering length $a_{K^{-} d}$ :

$$
\varepsilon_{1 s}+\frac{i}{2} \Gamma_{1 s}=2 \alpha^{3} \mu^{2} a_{K^{-} d}\left[1-2 \alpha \mu(\ln \alpha-1) a_{K^{-} d}+\ldots\right]
$$

The $K^{-} p s$-wave scattering length $a_{K^{-}} p$ is related to the $\bar{K} N$ isospin dependent $(\mathrm{I}=0,1)$ scattering lengths $a_{0}$ and $a_{1}$ through the average:

$$
a_{K^{-} p}=\frac{1}{2}\left[a_{0}+a_{1}\right]
$$

In order to obtain the individual isoscalar $a_{0}$ and isovector $a_{1}$ scattering lengths one has to measure also the kaonic deuterium, which provides information on a different combination of $a_{0}$ and $a_{1}$ :

$$
\begin{gathered}
a_{K^{-} n}=a_{1} \\
a_{K^{-} d}=\frac{4\left[m_{N}+m_{K}\right]}{\left[2 m_{N}+m_{K}\right]} Q+C
\end{gathered}
$$

where

$$
Q=\frac{1}{2}\left[a_{K^{-} p}+a_{K^{-} n}\right]=\frac{1}{4}\left[a_{0}+3 a_{1}\right] .
$$

The first term in (1.5) represents the lowest-order impulse approximation $\left(K^{-}\right.$scattering from each (free) nucleon of deuterium). The second term, $C$, includes all higher-order contributions, namely all other physics associated with the $K^{-} d$ three-body interaction.

The three-body system, $\bar{K} N N$, can be studied by solving Faddeev-type equations, taking into account the dynamics of the system, namely specifying the two-body interactions.

\section{The SIDDHARTA experiment at DA $\Phi N E$}

DA $\Phi N E$ (Double Annular $\Phi$ Factory for Nice Experiments) is a world-class electron-positron collider $[6,7]$ at the National Laboratories of Frascati (LNF) in Italy. DAФNE is a unique lowenergy kaon source via the decay of $\phi$-mesons produced almost at rest, which decay with a probability of $48.9 \%$ in $K^{+} K^{-}$, with a momentum of $127 \mathrm{MeV} / \mathrm{c}$, and a momentum spread $\Delta \mathrm{p} / \mathrm{p}<$ $0.1 \%$. This "kaon beam" is intensively used for studies of the low-energy kaon - nucleon/nuclei interactions, a field still largely unexplored.

The SIDDHARTA (Silicon Drift Detector for Hadronic Atom Research by Timing Application) experiment ended its data taking campaign in November 2009, after having performed kaonic atoms transitions measurements on the upgraded DA $\Phi$ NE collider. 
In the SIDDHARTA experiment the monochromatic low-energy charged kaons are degraded and stopped in a cryogenic gaseous target where kaonic atoms are efficiently produced.

Fig. 2 shows a schematic view of the SIDDHARTA setup, which consisted of three main components: the kaon detector, a X-ray detection system and a cryogenic target system.

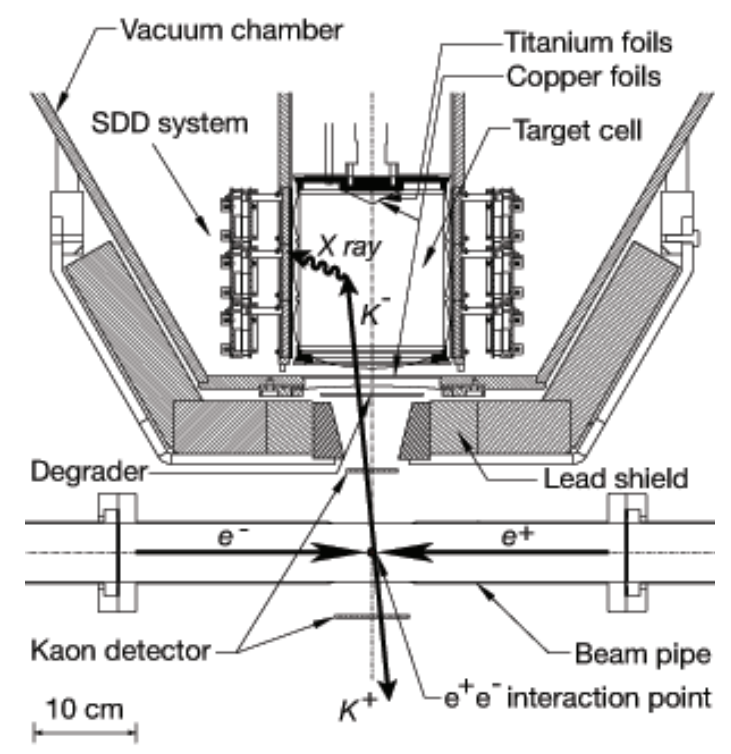

Figure 2: Schematic view of the SIDDHARTA setup [8].

The cryogenic gas-target system is a critical element of the experiment, because the yields of kaonic-atom X-rays decrease sensitively towards higher density due to collisions and Stark mixing with other atoms. For this reason it is is essential to employ a low density gaseous target. The cylindrical target cell, $13.7 \mathrm{~cm}$ in diameter and $15.5 \mathrm{~cm}$ in height, was located inside the vacuum chamber and it was filled with Hydrogen at $22 \mathrm{~K}$ and $\mathrm{p}=2.5$ bar.

The kaonic atoms X-rays were detected using the Silicon Drift Detectors (SDDs). For the first time, large area SDDs, characterized by good values of energy resolution (160 eV FWHM at $6 \mathrm{keV}$ ) and time resolutions (800 ns), which turned out essential for the background suppression, were developed as $3 \times 1 \mathrm{~cm}^{2}$ cells on one silicon wafer, for SIDDHARTA at DAФNE. A detailed description of the experimental setup is given in Ref.[9].

During the SIDDHARTA data taking campaign the following measurements were performed:

- kaonic hydrogen X-ray transitions to the $1 s$ level, which represents the most precise measurement ever [9].

- kaonic helium4 transitions to the $2 p$ level, the first measurement using a gaseous target [10], [11].

- kaonic helium3 transitions to the $2 p$ level, the first measurement ever [11], [8].

- kaonic deuterium X-ray transitions to the $1 s$ level - as an exploratory measurement [12].

The kaonic hydrogen spectrum together with the deuterium data used for background evaluation is shown in Fig. 3, where a global simultaneous fit of the hydrogen and deuterium spectra 
was performed. Fig. 3(a) shows the residuals of the measured kaonic hydrogen X-ray spectrum after subtraction of the fitted background. $K$-series X-rays of kaonic hydrogen were clearly observed, while those for kaonic deuterium were not visible [9]. This appears to be consistent with the theoretical expectation of lower X-ray yield and greater transition width for deuterium .

Fig. 3 (b) and (c) show the fit result with the fluorescence lines from the setup materials and a continuous background.

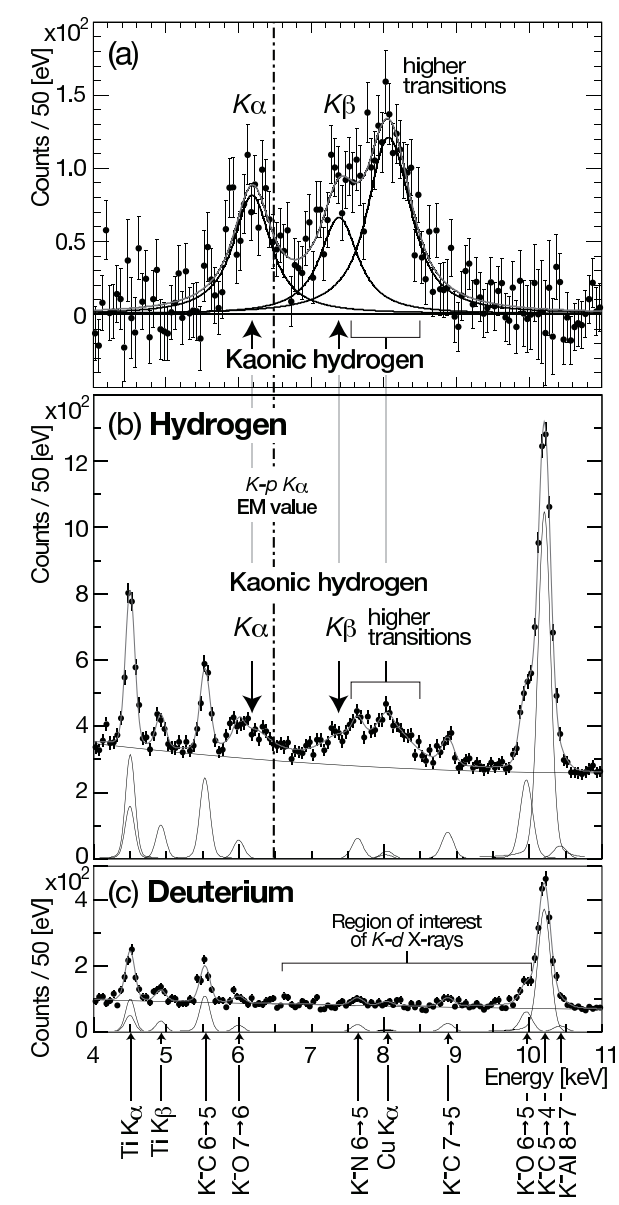

Figure 3: The global simultaneous fit of the $\mathrm{X}$-ray energy spectra of hydrogen and deuterium data. (a) Residuals of the measured kaonic-hydrogen X-ray spectrum after subtraction of the fitted background, clearly displaying the kaonic-hydrogen $K$-series transitions. (b)(c) Measured energy spectra with the fit lines. [9].

The vertical dot-dashed line in Fig. 3 indicates the X-ray energy of kaonic-hydrogen $K_{\alpha}$, calculated using the electromagnetic interaction only. Comparing the measured kaonic hydrogen $K_{\alpha}$ peak with the electromagnetic value, a repulsive-type shift (negative $\varepsilon_{1 s}$ ) of the $1 s$-energy level turned out.

Many other lines were detected in both spectra, as indicated with arrows in the figure. These kaonic atom lines result from high- $n \mathrm{X}$-ray transitions of kaons stopped in the target cell wall made of Kapton $\left(\mathrm{C}_{22} \mathrm{H}_{10} \mathrm{O}_{5} \mathrm{~N}_{2}\right)$ and its support frames made of aluminum. There are also characteristic $\mathrm{X}$-rays from the titanium and copper foils installed for calibration. 
As a result, the $1 s$-level shift $\varepsilon_{1 s}$ and width $\Gamma_{1 s}$ of kaonic hydrogen were determined to be [9]:

$$
\begin{aligned}
& \varepsilon_{1 s}=-283 \pm 36(\text { stat }) \pm 6(\text { syst }) \mathrm{eV} \\
& \Gamma_{1 s}=541 \pm 89(\text { stat }) \pm 22(\text { syst }) \mathrm{eV} .
\end{aligned}
$$

This is the most precise measurement performed so far of the $K$-series X-rays of kaonic hydrogen atoms.

The precise determination of the shift and width provides new constraints on theories, having reached a quality which demands refined calculations of the low-energy $\bar{K} N$ interaction.

For a deeper understanding of the $\bar{K} N$ interaction, it is essential to measure the kaonic-deuterium $\mathrm{K}$-series X-rays to disentangle the isoscalar and isovector scattering lengths. The SIDDHARTA experiment, after performing the most precise measurement on kaonic hydrogen, made the first exploratory study of kaonic deuterium, from which the $\mathrm{Kd} K_{\alpha}$ yield was extracted:

$$
Y\left(K_{\alpha}\right)=0.0019 \pm 0.0012
$$

Such values are compatible with what is expected, namely a yield of a factor about 10 smaller than the kaonic hydrogen yield, estimated to be between $1 \%$ to $2 \%$ for $K_{\alpha}$. The larger absorption in the $2 p$ state (larger $\Gamma_{2 p}$ ) in the case of kaonic deuterium atoms, as compared to kaonic hydrogen, causes the lower $K_{\alpha}$ yield. The Kd spectrum is shown in Fig. 4a.

From the data analysis, the upper limits for the yields turned out (CL 90\%): $Y\left(K_{t o t}\right)<0.0143$, $Y\left(K_{\alpha}\right)<0.0039[12]$.

Apart of the kaonic hydrogen and kaonic deuterium measurement, the SIDDHARTA experiment measured at the DA $\Phi$ NE electron-positron collider, both the $K^{-4} \mathrm{He}$ and the $K^{-3} \mathrm{He} 3 d \rightarrow 2 p$ transitions using gaseous targets $[10,8,11]$. The use of gaseous targets avoided the weakening of the yields due to the Stark effect and, also, the necessity to take into account the systematic uncertainty of the Compton scattering of X-rays inside the liquid helium target, which was big source of systematic errors in the previous kaonic helium-4 experiments.

Fig. 4 shows the X-ray energy spectra of different target data, (a) deuterium $(2.50 \mathrm{~g} / \mathrm{l})$, (b) helium-3 (0.96 g/l), (c) helium-4 (1.65 g/l), and (d) helium-4 (2.15 g/l). Several kaonic atoms Xray peaks are indicated for the deuterium target data. The X-rays of kaonic carbon $\left(K^{-} \mathrm{C}\right)$, kaonic nitrogen $\left(K^{-} \mathrm{N}\right)$, and kaonic oxygen $\left(K^{-} \mathrm{O}\right)$ were identified as produced from the Kapton polyimide used for the target cell and the target entrance window. The kaonic aluminum $\left(K^{-} \mathrm{Al}\right)$ was also seen. It comes from the target cell frame and the structures of the SDDs. For the data sets of helium-3 and helium- 4 targets, clear $\mathrm{L}_{\alpha}(3 d \rightarrow 2 p)$ peaks are seen. Other Balmer series X-rays can be also seen as smaller peaks. In the figure, the sum of the higher state transitions $(n>5)$ is indicated as $\mathrm{L}_{\text {high }}$.

Two data sets of helium-4 targets were merged. The systematic uncertainty was calculated as the quadratic sum of the individual contributions.

The obtained values for $\varepsilon_{2 p}$ and $\Gamma_{2 p}$ are $[10,8,11]$ :

$$
\begin{aligned}
& K^{-3} \mathrm{He}: \varepsilon_{2 p}=-2 \pm 2(\text { stat }) \pm 4(\text { syst }) e V \\
& K^{-4} \mathrm{He}: \varepsilon_{2 p}=+5 \pm 3(\text { stat }) \pm 4(\text { syst }) e V
\end{aligned}
$$




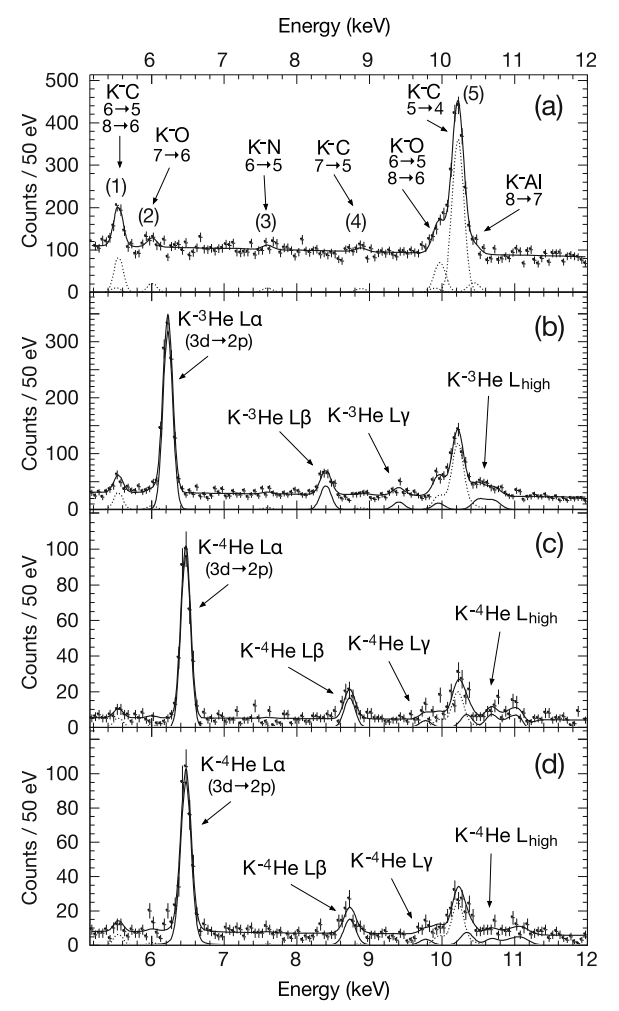

Figure 4: X-ray energy spectra of different SIDDHARTA targets data in the synchronous time window, (a) deuterium (2.50 g/l), (b) helium-3 (0.96 g/l), (c) helium-4 (1.65 g/l), and (d) helium-4 (2.15 g/l). The positions of the kaonic helium X-ray lines in (b), (c), (d) are indicated by arrows.

$$
\begin{aligned}
& K^{-3} \mathrm{He}: \Gamma_{2 p}=6 \pm 6(\text { stat }) \pm 7(\text { syst }) \mathrm{eV} \\
& K^{-4} \mathrm{He}: \Gamma_{2 p}=14 \pm 8(\text { stat }) \pm 5(\text { syst }) \mathrm{eV} .
\end{aligned}
$$

The $K^{-}{ }^{3} \mathrm{He}$ measurements were perormed for the first time. The $K^{-}{ }^{4} \mathrm{He}$ SIDDHARTA results for $\varepsilon_{2 p}$ with gaseous target are consistent, within the errors, with the results of the KEK-PS E570 [13] with liquid target.

\section{Kaonic deuterium: the SIDDHARTA-2 experiment}

The kaonic deuterium X-ray measurement represents the most important experimental information missing in the low-energy antikaon-nucleon interactions field.

The experimental challenge of the kaonic deuterium measurement is the very small kaonic deuterium X-ray yield (one order of magnitude less than for hydrogen), the even larger width and the difficulty to perform X-ray spectroscopy in the high radiation environments of the machines delivering kaons.

SIDDHARTA-2 is a new experiment, which will be installed on DAФNE in Sprimg 2019, taking advantage of the experience gained in the preceding SIDDHARTA experiment on kaonic hydrogen and kaonic helium. 
The goal of the new apparatus is to increase drastically the signal-to-background ratio, by gaining in solid angle, taking advantage of new SDDs with improved timing, and implementing additional veto systems in order to perform the kaonic deuterium X-ray measurement. The Fig.5 shows the SIDDHARTA-2 apparatus.

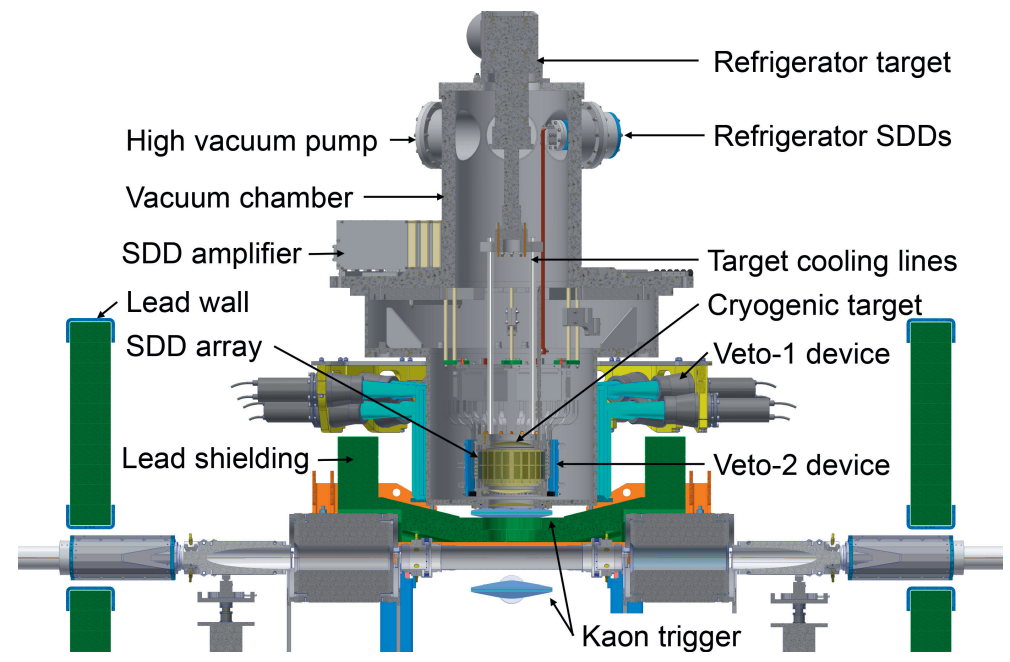

Figure 5: The SIDDHARTA-2 setup with the cryogenic target cell surrounded by the SDDs and the Veto-2 system within the vacuum chamber, while the Veto- 1 device is surrounding the vacuum chamber on the outside.

A detailed Monte Carlo simulation was performed within the GEANT4 framework to optimise the critical parameters of the setup, like target size, gas density, detector configuration and shielding geometry. The Monte Carlo simulation took into account all the improvements with the following assumptions: the values of shift and width of the 1s ground state of kaonic deuterium are -800 $\mathrm{eV}$ and $750 \mathrm{eV}$, respectively; yields ratios $\mathrm{K}_{\alpha}: \mathrm{K}_{\beta}: \mathrm{K}_{\text {total }}$ are those of kaonic hydrogen, with an assumed $\mathrm{K}_{\alpha}$ yield of $10^{-3}$. Fig. 6 shows the expected spectrum for an integrated luminosity of $800 \mathrm{pb}^{-1}$ delivered by DAФNE in similar machine background conditiond as in SIDDHARTA runs. The extracted shift and width can be determined with precisions of about $30 \mathrm{eV}$ and $80 \mathrm{eV}$, respectively, similar to the SIDDHARTA results for kaonic hydrogen.

\section{Conclusions}

The DAФNE collider delivers an excellent quality low-energy charged kaons beam. Such a beam was intensively used by the SIDDHARTA collaboration to perform unique quality measurements of kaonic atoms. Presently, an enlarged collaboration, SIDDHARTA-2, is upgrading the setup in order to perform the kaonic deuterium measurement. SIDDHARTA and SIDDHARTA-2 experiments on DA $\Phi N E$ collider provide unique quality results for the understanding of the lowenergy QCD in the strangeness sector, with implications going from particle and nuclear physics to astrophysics.

\section{Acknowledgments}

We thank C. Capoccia and G. Corradi, from LNF-INFN; and H. Schneider, L. Stohwasser, and 


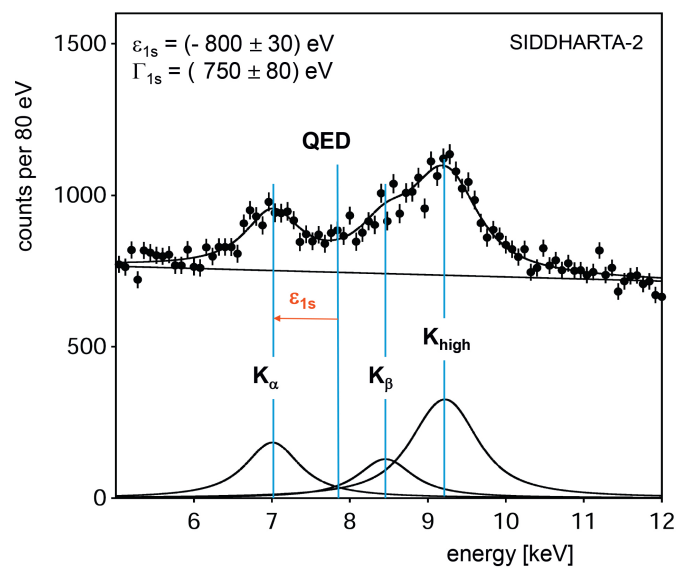

Figure 6: Simulated SIDDHARTA-2 kaonic deuterium spectrum, assuming a shift $\varepsilon_{1 s}=-800 \mathrm{eV}$ and width $\Gamma_{1 s}=750 \mathrm{eV}$ of the $1 \mathrm{~s}$ state, as well as a $\mathrm{K}_{\alpha}$ yield of $10^{-3}$. The spectrum was simulated for an integrated luminosity of $800 \mathrm{pb}^{-1}$.

D. Pristauz-Telsnigg from Stefan-Meyer-Institut, for their fundamental contribution in designing and building the SIDDHARTA setup. We thank as well the DAФNE staff for the excellent working conditions and permanent support. Part of this work was supported by the Austrian Science Fund (FWF): [P24756-N20]; Austrian Federal Ministry of Science and Research BMBWK 650962/0001 VI/2/2009; the Grantïn-Aid for Specially Promoted Research (20002003), MEXT, Japan; the Croatian Science Foundation, under project 1680; Minstero degli Affari Esteri e della Cooperazione Internazionale, Direzione Generale per la Promozione del Sistema Paese (MAECI), Strange Matter project; Polish National Science Center through grant No. UMO-2016/21/D/ST2/01155; Ministry of Science and Higher Education of Poland grant no 7150/E-338/M/2018.

\section{References}

[1] J. Zmeskal, Particle and Nuclear Physics 61, 512 (2008).

[2] S. Deser, M.L. Goldberger, K. Baumann, and W. Thirring, Phys. Rev. 96 (3), 774 (1954).

[3] T.L. Trueman, Nucl. Phys. 26, 57 (1961).

[4] U.G. Meißner, U. Raha, and A. Rusetsky, Eur. Phys. J. C 35, 349 (2004).

[5] U.G. Meißner, U. Raha, and A. Rusetsky, Eur. Phys. J. C 47, 473 (2006).

[6] C. Milardi et al., Int. J. Mod. Phys. A 24360 (2009).

[7] M. Zobov et al., Phys. Rev. Lett. 104174801 (2010).

[8] M. Bazzi et al., Phys. Lett. B 697 (3), 199 (2011).

[9] M. Bazzi et al., Phys. Lett. B 704, 113 (2011).

[10] M. Bazzi et al., Phys. Lett. B 681 , 310 (2009).

[11] M. Bazzi et al., Phys. Lett. B 714 , 40 (2012).

[12] M. Bazzi et al., Nucl. Phys. A 907 69, (2013).

[13] S. Okada, S. et al., Phys. Lett. B 653, 387 (2007). 